\title{
DIFFERENTIAL ROTATION IN STARS WITH CONVECTION ZONES
}

\author{
Peter A. Gilman \\ High Altitude Observatory \\ National Center for Atmospheric Research \\ Boulder, Colorado 80307/USA
}

\section{Introduction}

The topic I was originally assigned for this colloquium was "Generation of Non Thermal, Non 0scillatory Motions". Being basically a fluid dynamicist, at first I thought this meant I was supposed to talk about the origin of motions which are not thermally driven, i.e., I should not talk about convection. But then I realized all that was meant was that I was to talk about bulk fluid motions, rather than the molecular "thermal" motion of stellar gas that defines its temperature. Obviously the original question was posed by a stellar spectroscopist! Having surmounted that small semantic hurdle, I began to think about all the ways circulatory motions might be generated in a star. All manner of fluid dynamical instabilities come to mind--not only convective instability, but also barotropic or inertial, baroclinic, KelvinHelmholz, Rayleigh-Taylor, Goldreich-Shubert, Solberg-Hoiland, etc. The list is large, overlapping, I am sure confusing to an observer (and to many a theoretician). Then there are Eddington-Sweet currents, and several additional motions arising from the presence of magnetic fields--fields which give rise to magnetic buoyancy of flux tubes, and large collection of magnetohydrodynamic instabilities.

I could try to review all of these effects in this talk--but that has been done in several other places, for example in the recent book Rotating Stars by Tassoul, and a soon to be published book on astrophysical magnetohydrodynamics by Parker. The audience is better advised to look there. In addition, I am not convinced that an observer can gain much guidance on how to interpret his stellar spectra from extrapolations that predict the nonlinear consequences of these instabilities (with the exception of convection). With the limited time that I have, I would instead like to address a single question: "What differential rotation should we expect to find in a star that ratates and has a convection zone?"

thy this particular question? Aside from convection itself, differential rotation is probably the largest amplitude motion to be found in a typical stellar photosphere. The star's average rotation is most likely larger still (and easier to measure) but solid rotation in and of itself is fluid dynamically trivial; how it affects other motions to produce differential rotation is, I hope to convince you, very interesting.

Why consider only stars which have convection zones? I personally feel on safer ground for this case, but also I think more definite things can be said. Convection influenced by rotation can come to some sort of statistical equilibrium with the differential rotation it drives, and therefore the differential rotation can be expected to be present over a large fraction of the star's lifetime. By contrast, many of the other instabilities I mention may produce transients in the star, not seen 
again for a long time because the energy source for the instability is not replenished as fast as for convection (and maybe never). Ihat we are after, then, are statements about differential rotation forced by convection, that might be applicable to a wide variety of stars. All stars rotate, and all stars that convect will have differential rotation. The real question is how much and what kind.

Some further statements are perhaps in order. Differential rotation is from a fluid dynamical point of view a very important quantity to know about a star. It is probably the key to guessing its overall grobal dynamics. It may tell us what kind of dynamo action the star is experiencing--whether it should be undergoing cycles in its magnetic field. For example, it would be extremely useful to know the rotation and differential rotation of stars for which 01 in wilson sees calcium emission cycles.

Let me acknowledge before I get any further that I know measurements of differential rotation in stars are extremely difficult to achieve, and probably impossible for several classes of stars by present techniques. Measuring rotation itself is bad enough. In the end, it may turn out that we have to be content with predictions of differential rotation for many stars, guided by other information such as rotation, spectral type, abundance, etc.

\section{Approach to the Problem}

Let me first list some of the physical parameters and processes we might expect to be important in determining the differential rotation at the surface of a convecting star. Then I will review some of the models that have been developed to explain differential rotation in the sun, including some of the problems as well as successes. I have worked on models of this type myself, and I will summarize some of my own results which I think are relevant to the question I have posed. From these results I will ther extrapolate to some qualitative statements about other stars--main sequence stars mostly, but red giants will also be considered.

\section{Important Parameters and Processes}

\section{A. Convection Zone Parameters}

Probably the most important single parameter determining what kind of differential rotation to be expected in a stellar convection zone is the ratio of turnover time for the convection, to rotation time for the star. This determines how much rotation affects convection, introducing preference for certain convection patterns, as well as correlations between different velocity components implying nonlinear Reynolds stresses. The larger is this ratio, the more influence rotation has, because as the fluid element moves, coriolis forces have more time to act on it. We will iqnore systems with rotation so rapid that the departures from spherical geometry become of order unity.

In addition, certain other parameters are important, for example, convection 
zone depth, as a fraction of the radius. It is reasonable to expect that stars with shallow convection zones will have different patterns of differential rotation than stars with deep zones. The scale of the dominant convective modes will certainly be larger for deeper zones, and in general, the rotational influence will be stronger, because the turnover time is longer. The particular form the stratification of the zone takes will also be important--how may scale heights it contains as well as how superadiabatic the temperature gradient is. These quantities clearly relate back to the turnover time estimates, as does the total heat flux being carried through the convection zone.

\section{B. Physical Processes Affecting Angular : lomentum Distribution}

Any process that can redistribute angular momentum in a convection zone is potentially important for determining the differential rotation profile. Since angular momentum can be convected by the fluid itself, any circulation that has a component in either the radial or latitudinal direction, can change the rotation profile in the meridian plane. Conventionally, these circulations are broken into two tyoes, axisymmetric meridional circulation, and global departures therefrom, usually called eddies. The eddies produce net momentum transport when the east-west velocity in them is correlated with either the north-south flow, the radial flow, or both. This correlation is induced by the presence of the Coriolis force and is called a Reynolds stress. Molecular and small scale turbulent diffusion is swamped by the turbulence. The distinction between small scale turbulent diffusion and global eddy transport is an arbitrary one, the former usually representing transport of momentum by those motion scales not resolved in the particular model, the latter being explicitly calculated. Other azimuthal torques, such as produced by electromagnetic body forces, may also be present, but their effect has not generally been taken into account. Buoyancy forces, since they act in the radial direction, do not directly contribute azimuthal torques that can change the differential rotation, but of course they drive motions which transport momentum. Similarly, azimuthal pressure torques average out when integrated around a complete latitude circle.

Figure 1 illustrates angular momentum transport by different circulations. At the top, two schematic meridional circulations are shown, of opposite sense. If either pattern started up in a fluid originally in solid rotation and no other transport processes were acting, high latitudes and deeper layers would tend to speed up, relative to low latitudes and shallow layers, because the circulation would conserve angular momentum. The fluid would move toward a state of constant angular momentum. But now if sufficient diffusion is also present to link different layers and keep the angular velocity more nearly constant, the circulation on the left may produce equatorial acceleration, because fluid moving toward the equator on the outer branch, for example, crossing the dashed line, would contain more angular momentum than fluid moving toward the pole underneath, where the moment arm is shorter. 
Competing Mechanisms of Angular Momentum Transport
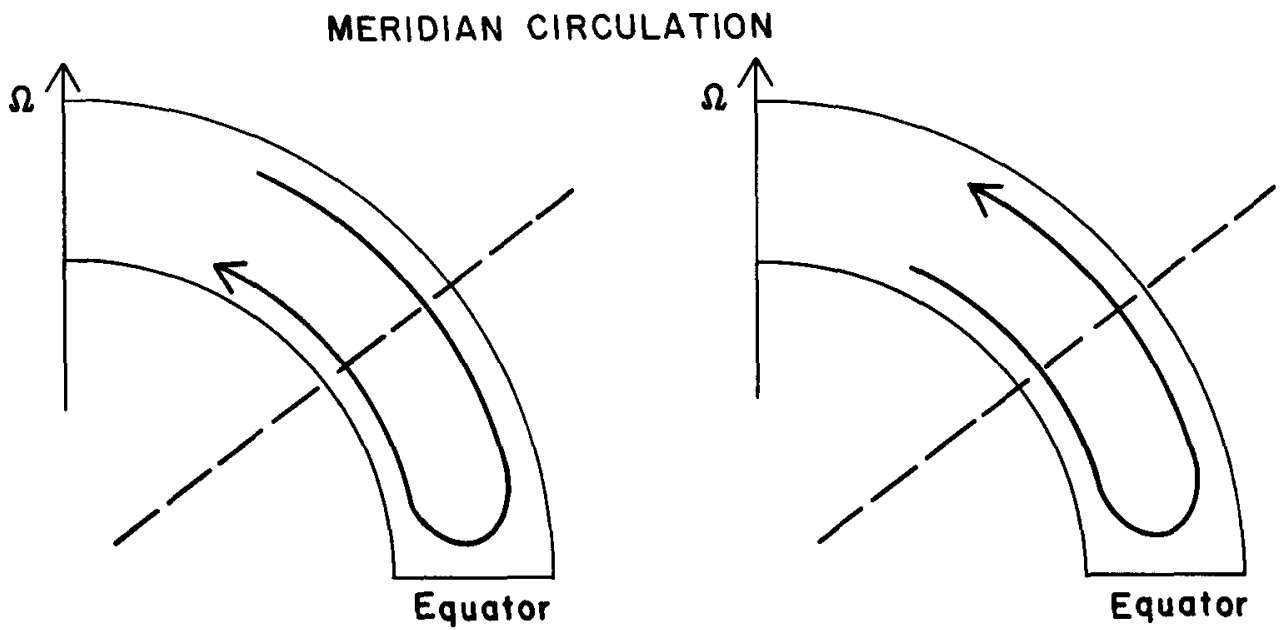

REYNOLDS STRESSES
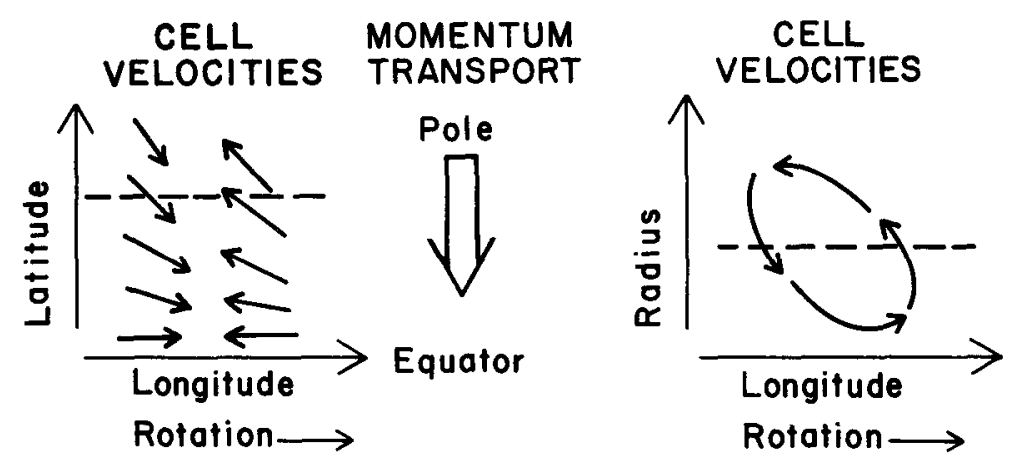

MOMENTUM TRANSPORT

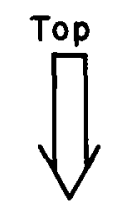

Bottom

DIFFUSION

In simplest (isotropic) form, tends to equalize angular velocities. May couple with meridian circulation to produce equatorial acceleration.

Figure 1. Angular momentum transport by circulation. 
On the other hand, Reynolds stresses will transport momentum toward the equator to drive an equatorial acceleration if the convection patterns adopt a horizontal flow pattern as seen in the left middle schematic. Fluid particles moving eastward in the direction of rotation have a component toward the equator; particles moving west are also moving poleward. If we average along the dashed line, we get a net momentum flux toward the equator, even if there is no net mass flux. In the right middle schematic, we see a pattern of circulation in longitude and radius which leads to a net flux of momentum inwards. The two patterns I have shown are in fact the most common ones seen in actual model calculations.

\section{Models Used in Solar Case}

Which angular momentum transport process dominates in producing differential rotation can only be determined by actual nonlinear model calculation. A number of such calculations have been carried out, mostly to explain solar equatorial acceleration.

One group of these models has been axisymmetric, in which all effects of convection are condensed into a few ad hoc parameters. In one such model, an anisotropic turbulent viscosity is assumed, which results in a meridian circulation qualitatively like that at the top left in Figure 1. With suitable choice of the sign and degree of anisotropy, the observed equatorial acceleration can be reproduced. This approach originated with Biermann (1951) and was exploited by Kippenhahn (1963), Cocke (1967) and in greater detail by Kohler (1970). The problem with it is that there is no real way to choose the sign and magnitude of anisotropy, without resort to fitting the correct differential rotation. Also, no account is taken of the influence of rotation upon the parameterization of the convection. Another approach which has been carried further is to assume the convective heat flux is weakly perturbed by rotation, such that it becomes a function of latitude. This was done by Durney and Roxburah (1971) and later developed further by Belvedere and Paterno (1976, 1977, 1978). Aqain the meridian circulation and convective parameterization are fitted to the observed differential rotation. The calculations are heavily dependent on the assumption of weak influence of rotation upon convection, which is not likely to be valid in the deep part of the solar convection zone. Also, there is no independent evidence that the heat flux parameterization is valid even under the assumptions made.

As you probably have already guessed, I do not believe these models are the correct ones for the sun, mostly because I suspect the parameterizations upon which they depend are grossly inaccurate. I prefer models in which the dynamics responsible for giving the correct differential rotation are explicitly calculated, with parameterizations of unresolved motions relegated to a less critical role, so their detailed form is less important. Such models have been developed, so far for physics considerably simpler than the real sun, but nevertheless instructive. In particular, 
models have been developed for nonaxisymmetric convection of a stratified liquid in a rotating spherical shel1. Early, mostly linear analyses by Busse $(1970,1973)$, Durney $(1970,1971)$ Kato and Yoshimura (1971) and Gilman (1972, 1975) demonstrated the preference for convective modes which transport momentum toward the equator, via the Reynolds stress mechanism described above. More recent, nonlinear calculations by myself (Gilman, 1976, 1977, 1978, 1979) have exploited this fact to determine in detail when equatorial acceleration occurs and with what amplitude relative to the convection which drives it and the basic rotation rate.

I will summarize some results obtained from those calculations. I will give only the most basic, qualitative effects, since the model is incompressible, and many details will change in the compressible case--which we are currently coding. There are other weaknesses of the model, such as larger than observed differentials in heat flux, which I cannot describe in detail, but which we expect to be improved in the compressible case.

\section{Summary of Relevant Results from Calculations of Convection} in a Rotating Spherical Shell

A. Finite amplitude equatorial acceleration is produced only when the influence of rotation upon convection is strong, i.e., the rotation time is less than the turnover time for convection. Under these circumstances, the angular velocity also decreases inward with depth; when the rotational constraint is very strong, the angular velocity predicted is nearly constant on cylinders concentric with the axis of rotation. If the rotation time is a few orders of magnitude shorter than the turnover time, then the latitudinal profile of differential rotation may be more complicated, but this is an unlikely case for stellar application.

B. When the convection zone is deep, say $1 / 3$ of the radius or more, the equatorial acceleration profile with latitude is broad, with essentially monotonic decrease in angular velocity to the poles.

c. When the convection zone is shallow, say 20 percent or less, and the rotational influence is strong, the angular velocity reaches a minimum in mid latitudes, and then increases again toward the poles. The width of the equatorial acceleration is determined by the depth of the layer.

D. For both deep and shallow convection zones, with weaker rotational influence (increased convective velocities) the profile switches from equatorial acceleration to deceleration. Angular velocity now increases with depth. There is an intermediate stage in which the angular velocity is highest in mid latitudes and lower near the equator and near the pole, while still decreasing with depth.

E. The maximum differential rotation sustainable by the convection is about 40 percent of the average rotation. For larger values, the feedback from the shear on the convection is strong enough to change the dominant patterns and consequently the 
differential rotation profile, resulting in a new equilibrium with lower amplitude differential rotation.

F. The maximum differential rotation maintainable by the convection has about the same kinetic energy in it as the convection itself. Larger differential rotation may be possible locally in the compressible case.

G. Amplitudes of individual convective modes may change radically with time while the differential rotation amplitude changes hardly at all.

The above statements concerning the profile of differential rotation for deep and shallow convection zones are summarized schematically in Figures 2 and 3 respectively. The extra bumos in the profiles for the shallow layer are intended to indicate that for a shallow layer, the convection, being smaller in horizontal scale itself, should produce more fine structure in the differential rotation profile.

In addition, we can say that first results from linear calculations of compressible convection in a rotating spherical shell indicate that moderate stratification (say a factor of 20 drop in density across the convection zone) wi11 not change any of these results significantly. Larger density variations will produce larger changes, but the qualitative statements we have made with respect to differential rotation with latitude we do not expect to change a great deal. But clearly such conclusions must be tested with a compressible model.

\section{Extranolation to the Convection Zone of a Star}

How do we extrapolate the above results to the convection zone of a star, which, after all, is generally a long ways from being a Boussinesq liquid? In the case of the sun, the theory is generally thought to apply to the deepest layers of the convection zone, which are more nearly Boussinesq, for which the natural scale for convection is nearly global. As filman and Foukal (1979) have argued, the small scale but large amplitude granules and super granules are most likely not responsible for the latitude gradient of rotation at all, but perhaps the radial gradients in the shallowest layers. In addition, these small scale motions appear to obscure the global convection or "giant cells" (Simon \& Weiss, 1968) as they are sometimes called.

A similar situation probably occurs in convection zones of other stars, as summarized in Figure 4. The little cells at the top are weakly influenced by rotation, and by themselves would produce a weak equatorial deceleration. But they sit on top of slower, larger scale convection below, which is much more strongly influenced by rotation, where the latitude gradient is really produced. This gradient of deep origin is then transmitted to the surface by the small cells.

One conclusion that can be drawn from this argument is that using surface turbulent velocities, to estimate the turnover time for the convection zone would lead to a gross under-estimate, therefore too smal1 a rotational influence and the wrong differential rotation. A better procedure, still very approximate, would be to esti- 
mate the turnover time from a mixing length model applied to the whole convection zone. This brings us to a prescrintion for estimating the differential rotation to be expected in certain main-sequence stars.

What we have done is to estimate convection zone depths and turnover times near the bottom of the zone, using a stellar envelope code, and then compare these with observed or estimated rotation times calculated from the well known summary of stellar rotation, by Kraft (1967). Part of this procedure is similar to one employed by Durney and Latour (1973) for another purnose, which calculation I discovered as I was beqinning these calculations. In fact, I used Latour's envelope code, which in turn is similar to an earlier one applied to estimate convection zone depths by Baker (unpublished). I chose essentially Baker's values for a sequence of stars from A to early $k$, the luminosity, radius, and effective temperature for which are shown in Fiqure 5. We then used these values, together with a composition of $X=.70, Y=.27$, $Z=.03$ in Latour's code to estimate convection zone depth for these stars, shown in Figure 6 , given as a function of $\alpha$, the ratio of assumed mixing length to pressure scale heiqht. The results are quite similar to those of Baker, though not identical. As expected, convection zone depth shrinks as a fraction of stellar radius as the mass and luminosity increase. Depending on the $\alpha$ choser, the convection zone effectively shrinks to zero somewhere between early $F$ and late $A$. Larqer $\alpha$ implies deeper convection zone.

Then, following Durney and Latour (1973) we find a convective velocity in meters/ second one pressure scale height up from the bottom, shown in Fiqure 7 , which we use to compute a turnover time $=$ scale height/velocity, shown in Figure 3 . ilote that it goes from $1-2 \times 10^{6} \mathrm{sec}$. for $G$ and late $F$ stars, down quickly to $10^{4} \mathrm{sec}$. and less by F?.

But earlv stars generally rotate much faster too, so the rotation time drops rapidly with increased mass, as seen in Figure 9, derived from Kraft (1967). (Here, for stars later than the sun, we assume an unper limit of the same angular velocity as the sun.) So how does the ratio change? This is plotted in Figure 10. We see that for $\alpha=1.0$, the sun is already in the steep part of the curve, with convection in slightly later stars much more strongly influenced by rotation, convection in slightly earlier stars much less so. For larger $\alpha$, earlier stars out to FO-F2 are as strongly influenced, or more so, by rotation than the sun is. 1:Ihich $\alpha$ is most reasonable (if any)? For the sun, $\alpha=1$ yields by my earlier arguments too shallow a convection zone $(<20 \%)$ to sustain an equatorial acceleration with angular velocity decreasing all the way to the pole, so I favor a larger $\alpha$ (at least 2). Suppose we take $\alpha=2$. Then from all our results we should expect broad equatorial acceleration on stars later than the sun, (unless they have much lower rotation rates than the sun) and also for slightly earlier stars, with an increasingly narrow equatorial acceleration out to $\log$ $! 1 /: 1_{0} \approx .12$. Maximum amnlitude in this equatorial acceleration would be about $40 \%$ of 


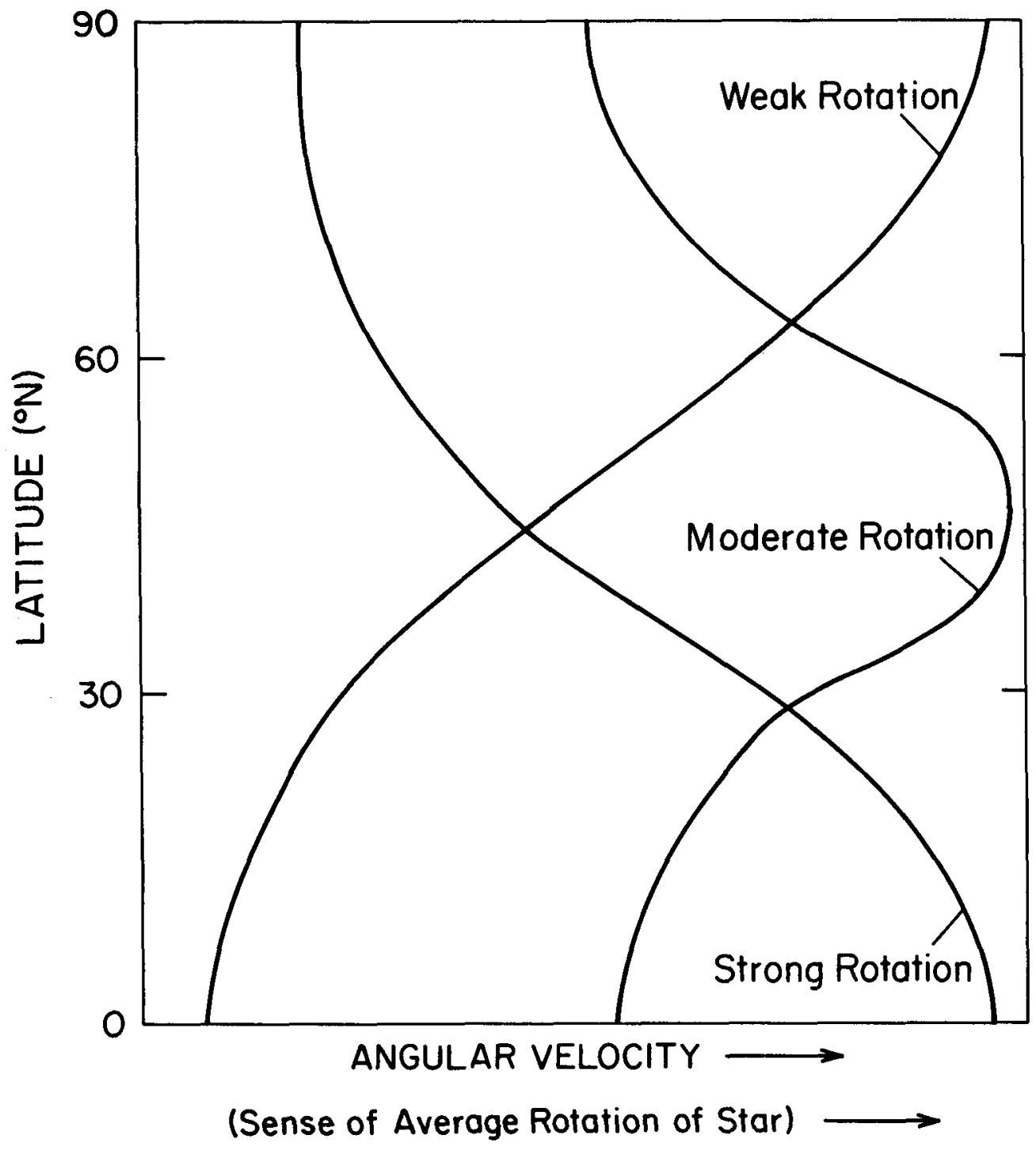

Figure 2. Qualitative schematic of differential rotation regimes for a deep convection zone. 


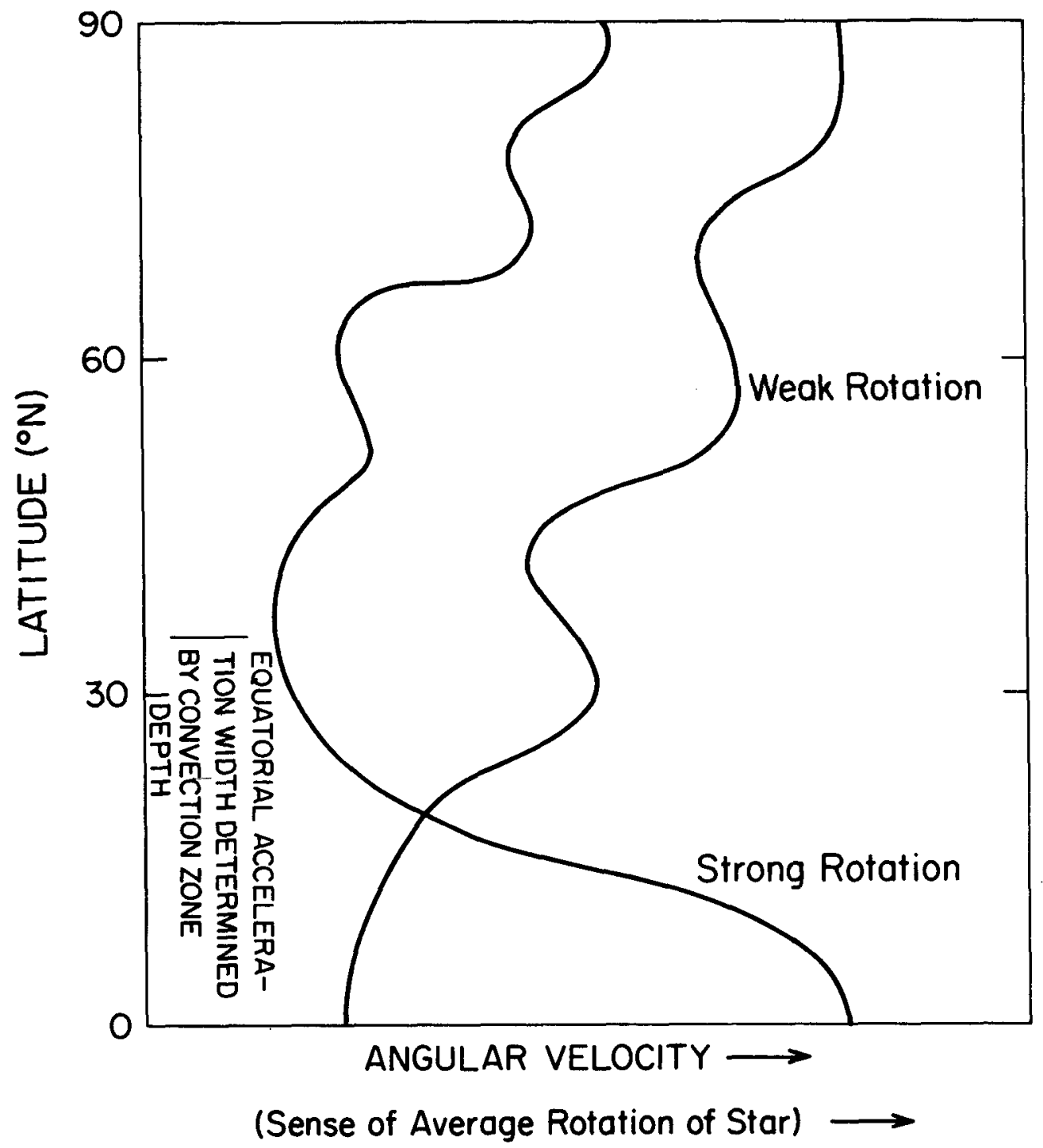

Figure 3. Qualitative schematic of differential rotation regimes for a shallow convection zone. 


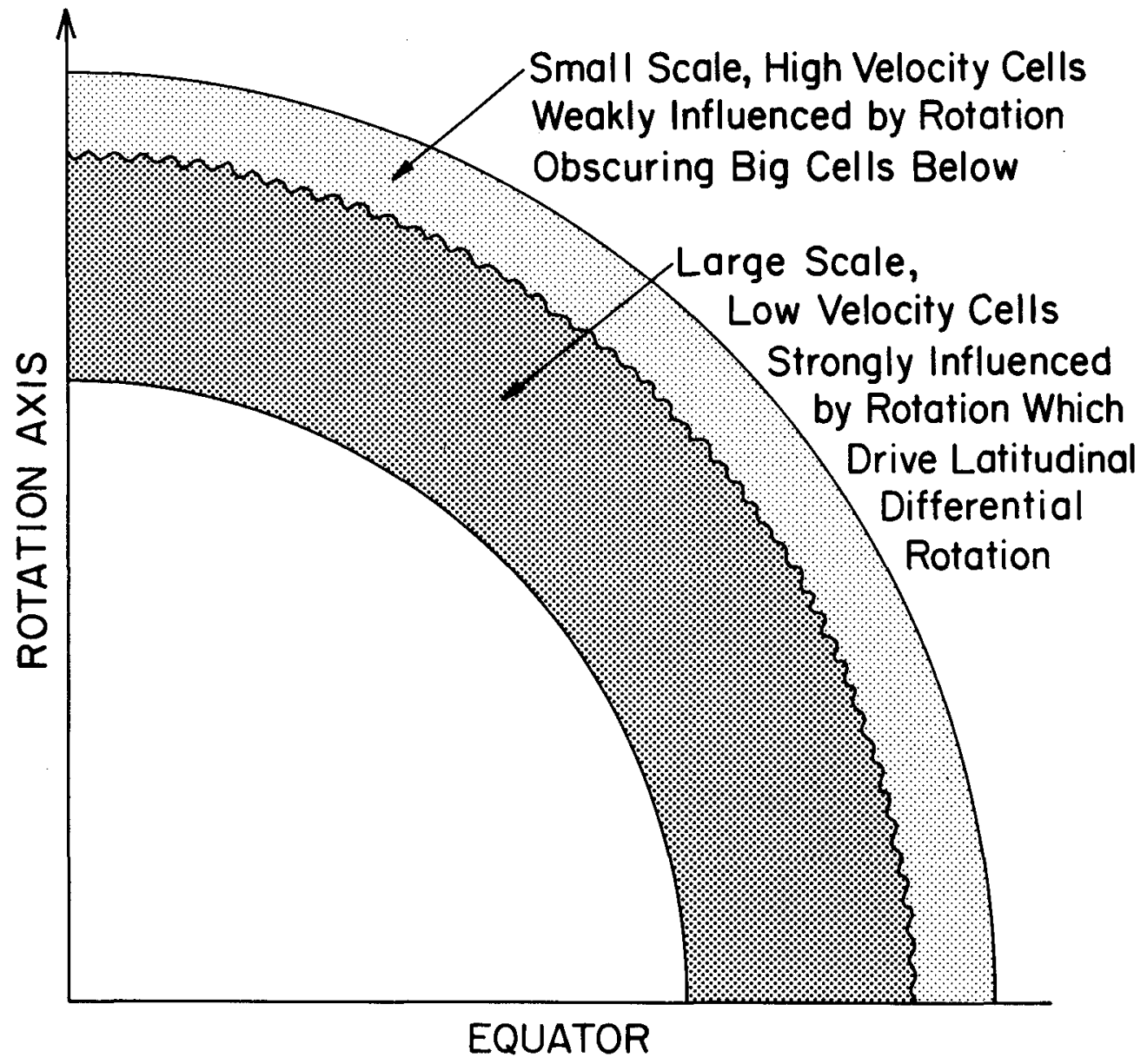

Figure 4. Schematic of a stellar convection zone. 
SPECTRAL TYPE

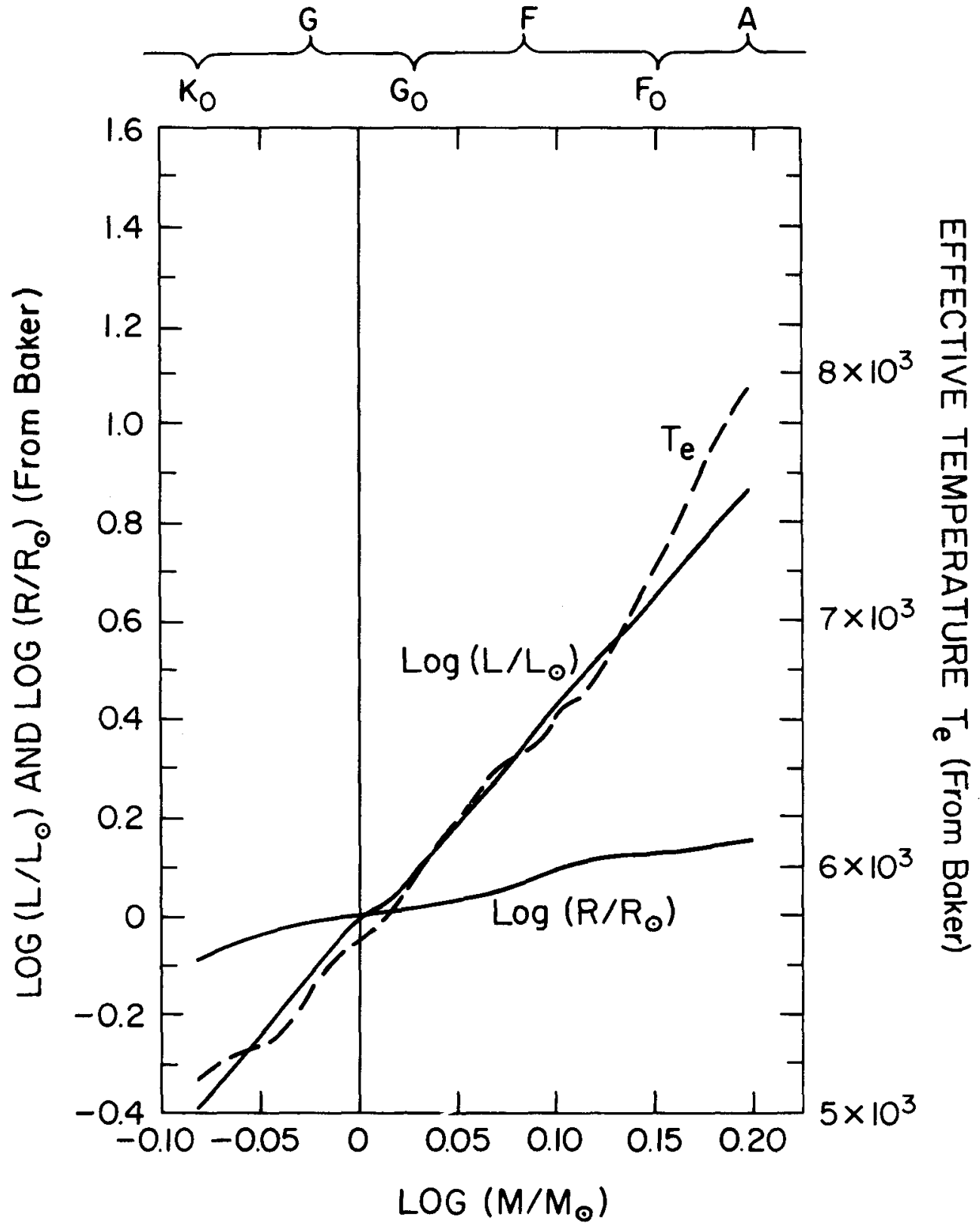

Figure 5. Relations between luminosity, radius, and effective temperature. 
SPECTRAL TYPE

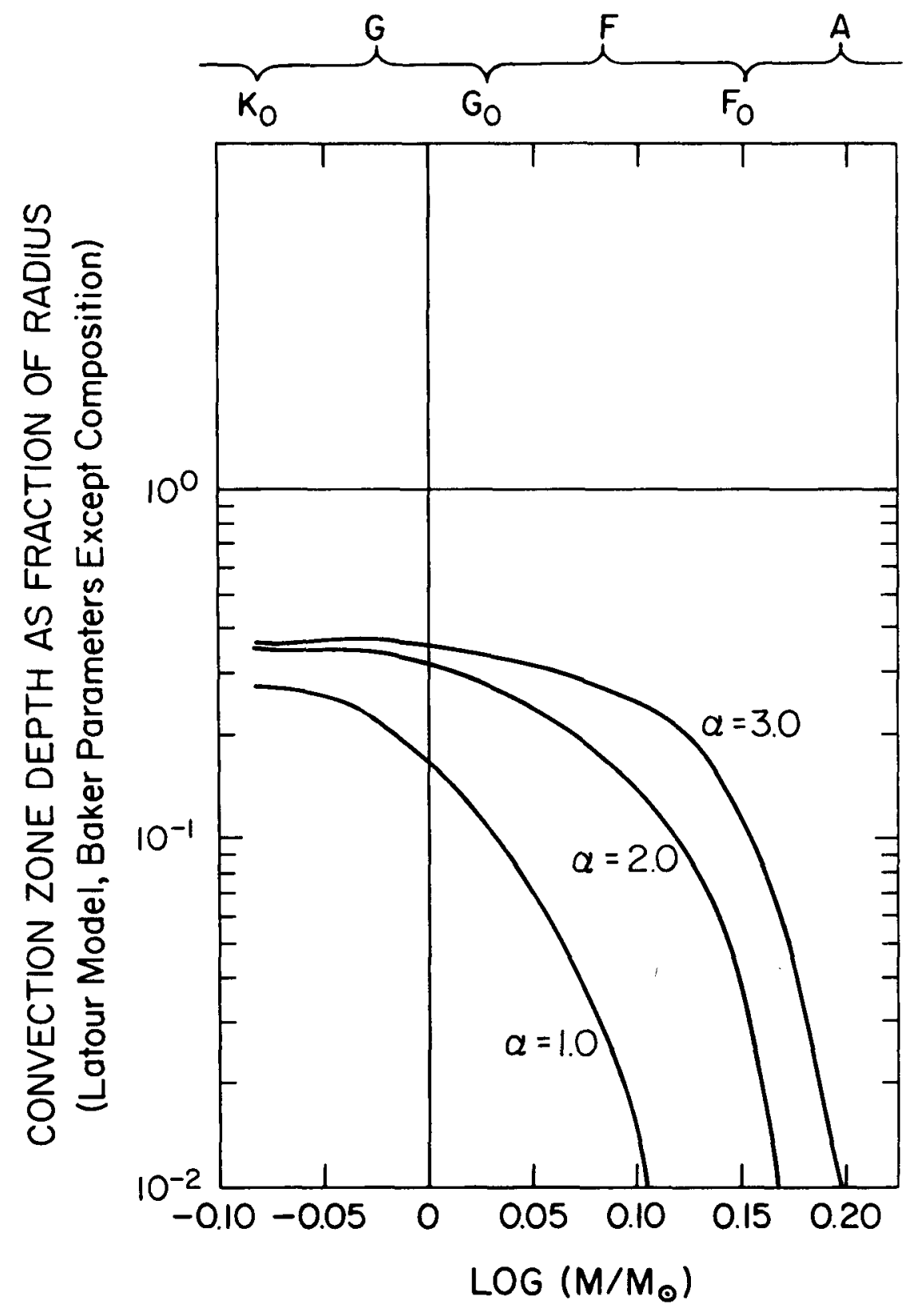

Figure 6. Convection zone depths. 
SPECTRAL TYPE

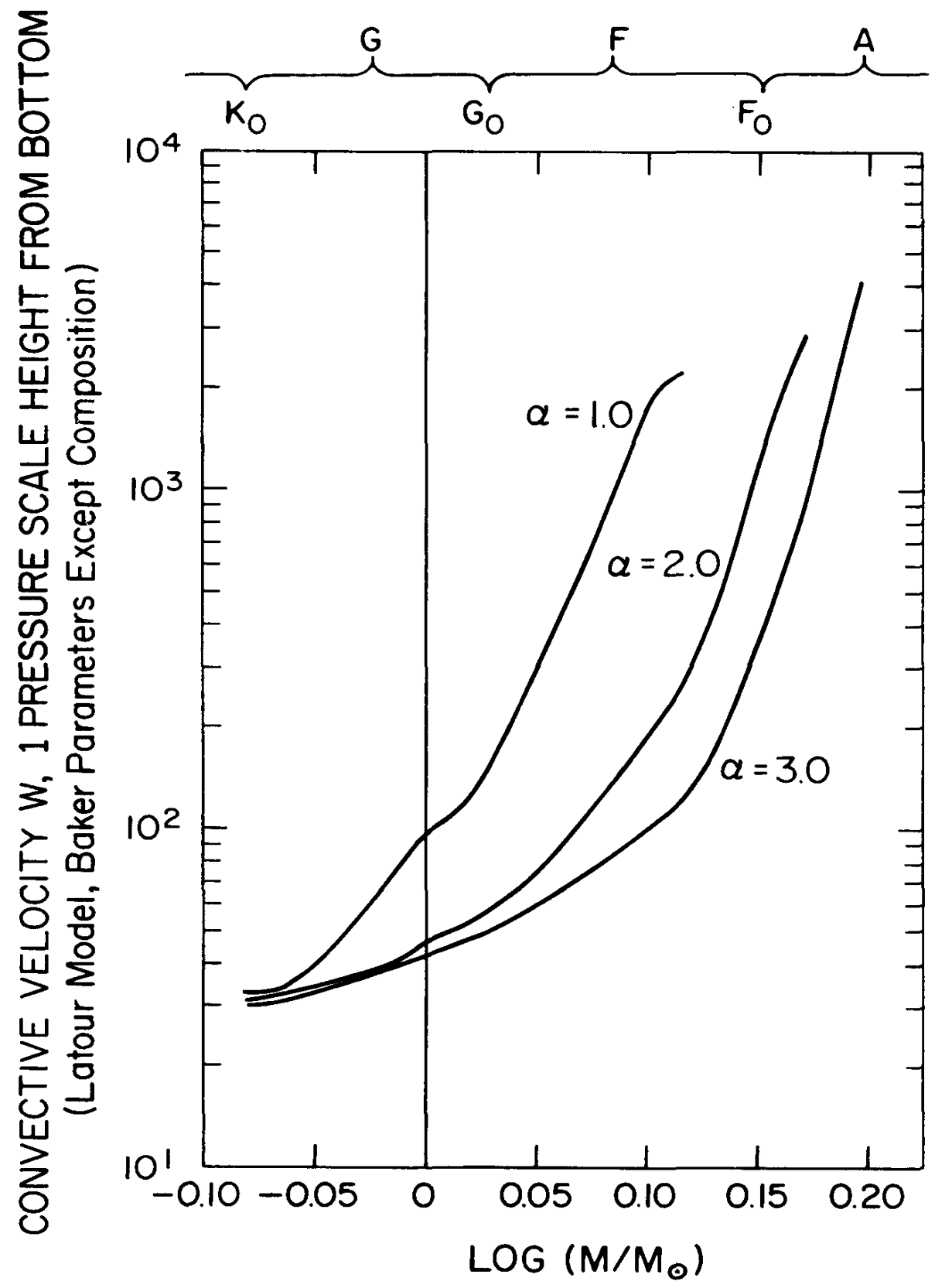

Figure 7. Convective velocities. 
SPECTRAL TYPE

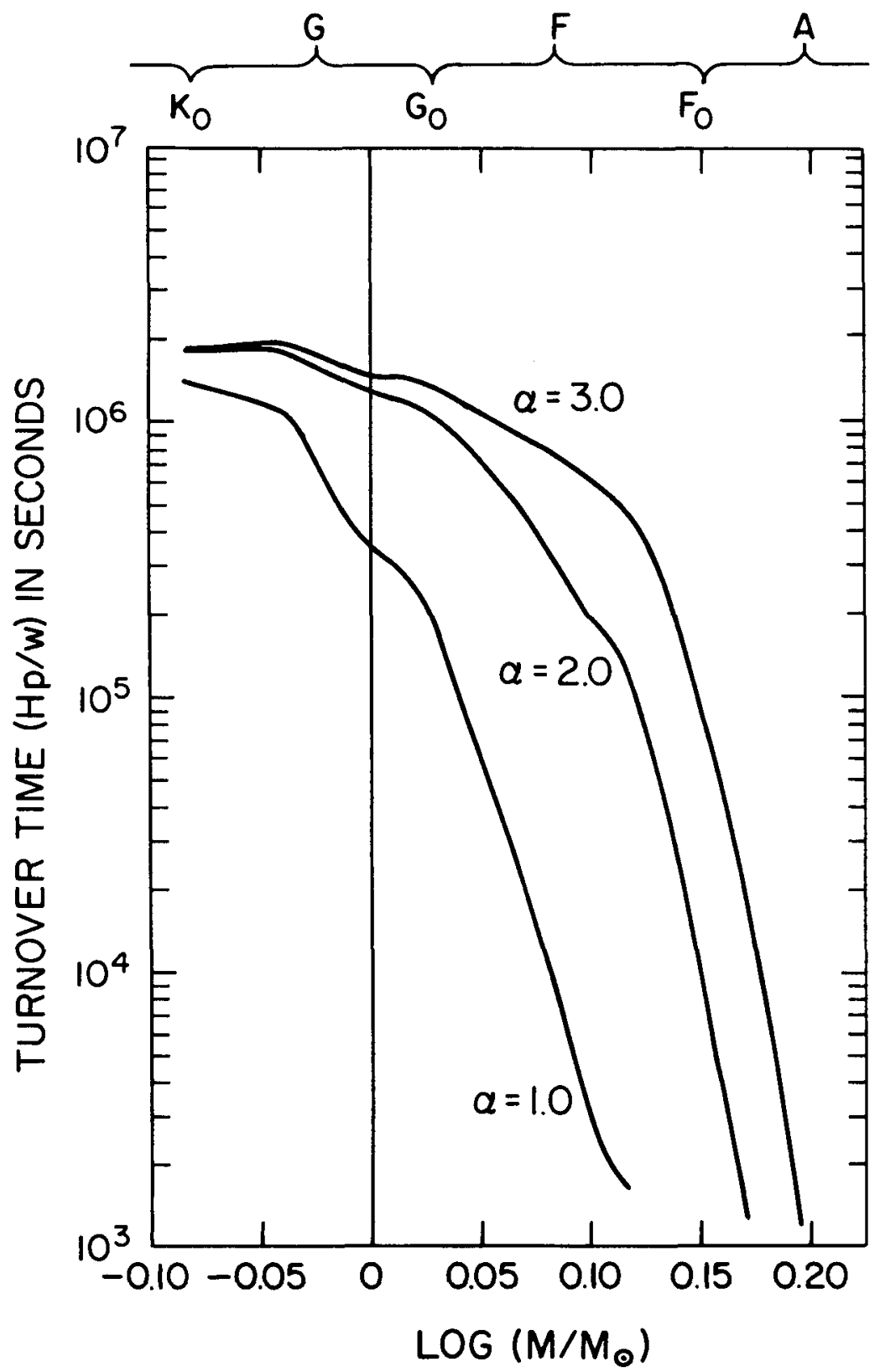

Figure 8. Convective turnover times. 
SPECTRAL TYPE

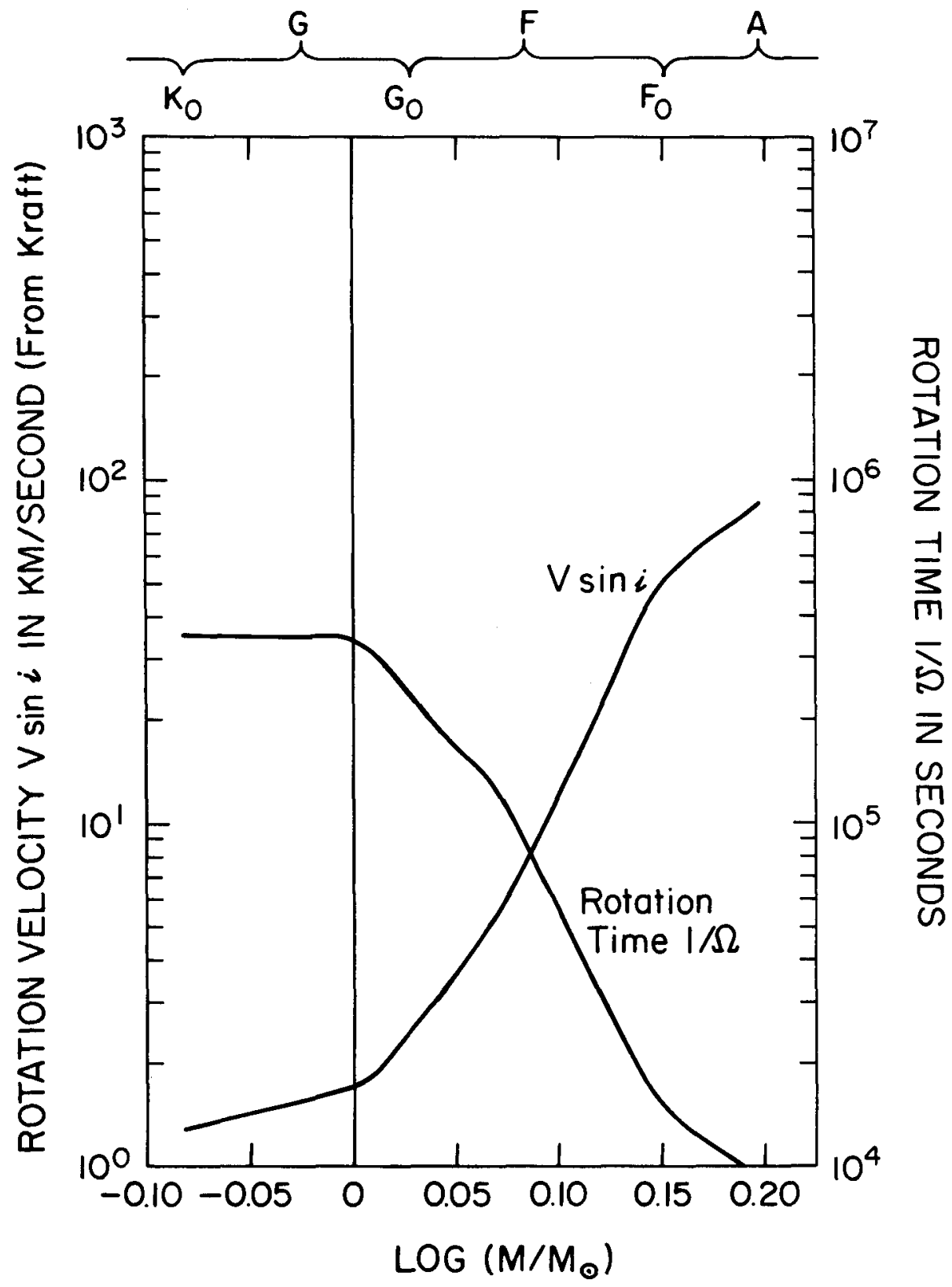

Figure 9. Rotation velocity and rotation time as a function of mass. 


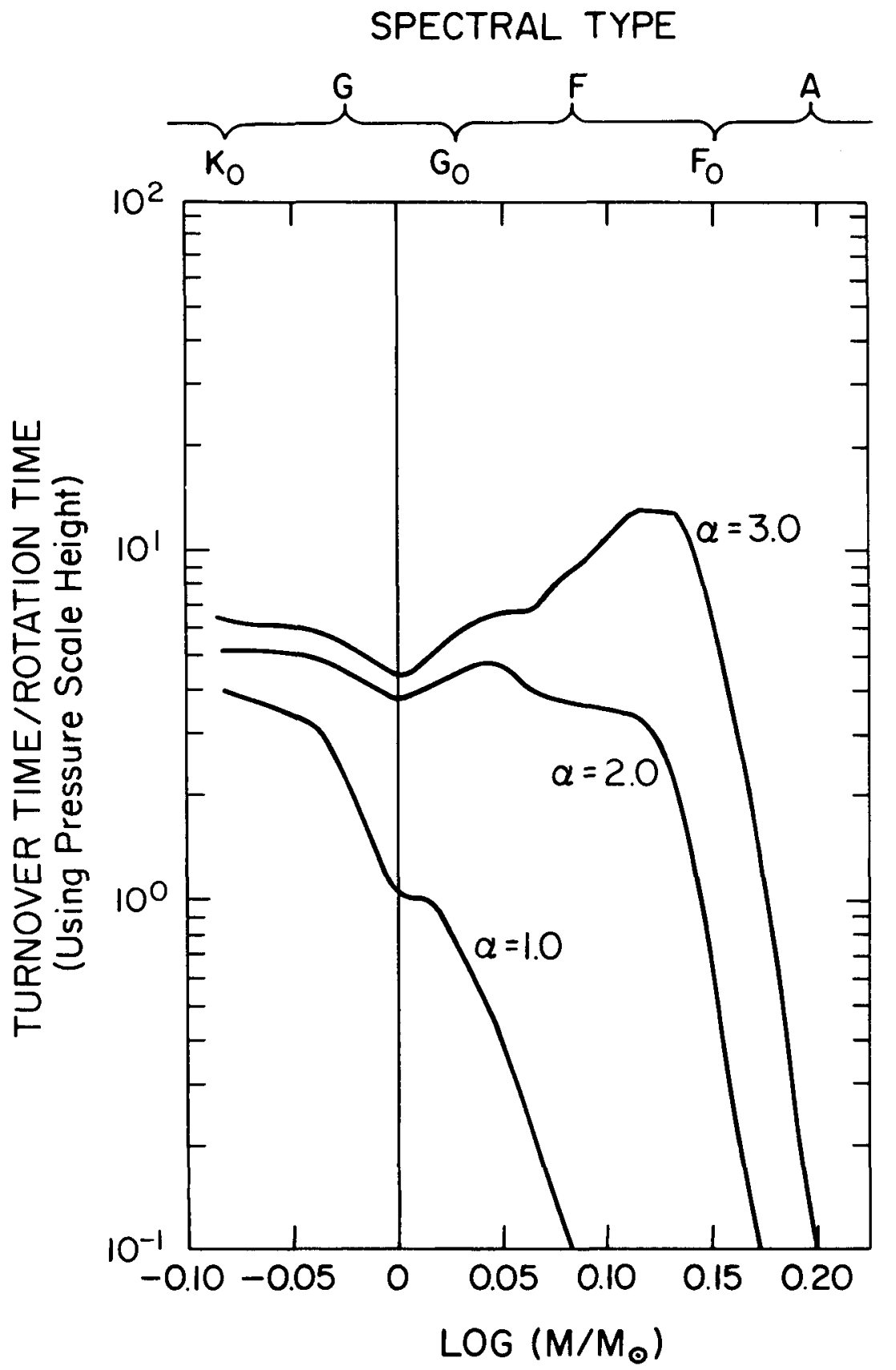

Figure 10. The ratio of turnover time to rotation time as a function of mass. 
the average rotation rate. Beyond that, there would be a rapid transition to equatorial deceleration, with a lot of structure in the profile, due to the dominance of small scale modes in the shallow zones there. The conclusion for $\alpha=3$ would be similar.

It would seem that rather analogous arguments could be made for red giants. Those with deep convection zones should have either broad equatorial accelerations or decelerations, depending on their rotation rate. Perhaps the calculations from which these inferences are made apply better here than for main sequence stars, since in most red giants the scale height is a large fraction of the convection zone depth so the convection is more nearly like that in a stratified liquid.

In closing, let me remark again that if one knows the rotation rate of the star, and a few other basic properties, one may be able to estimate its differential rotation even if one cannot observe it. For stars with surface emission features, from which an independent estimate of rotation rate, and perhaps even differential rotation, can be made, we may be able to test our conclusions in detail.

I close with a question for the observers: Can you tell the difference by spectral measurements between a star in solid rotation, and one with nearly the same surface average angular velocity that in fact is rotating differentially?

Acknowledgments. I thank Jean Latour for making available to me his stellar envelope code, and my student, Gary Glatzmaier, for running a number of calculations with this code for me. Dimitri !'ihalas was kind enough to read the manuscript for me. Previous discussions with Douglas Gough on this topic have been useful.

The ilational Center for Atmospheric Research is sponsored by the National Science Foundation. 


\section{References}

Belvedere, G. and Paterno, L., 1976: Solar Phys. 47, 525.

Belvedere, G. and Paterno, L., 1977: Solar Phys. 54, 289.

Belvedere, G. and Paterno, L., 1978: Solar Phys. 60, 203.

Biermann, L., 1951: ‥ Astrophys. 28, 304.

Busse, F., 1970: Astrophys. ‥ 159, 629.

Busse, F., 1973: Astron. Astrophys. 28, 27.

Cocke, 1.J., 1967: Astrophys. J. 150, 1041.

Durney, B.R., 1970: Astrophys. J. 161, 1115.

Durney, B.R., 1971: Astrophys. J. 163, 353.

Durney, B.R. and Latour, J., 1973: Geophys. Astrophys. Fluid Dyn. 9, 241.

Durney, 3.R. and Roxburgh, I.H., 1971: Solar Phys. 16, 3.

Gi7man, P.A., 1972: Solar Phys. 27, 3.

Gilman, P.A., 1975: ‥ Atmos. Sci. 32, 1331.

Gilman, P.A., 1976: in IAU Symposium \#71, Basic Mechanisms of Solar Activity, ed.

V. Sumba and J. Kleczek (Dordrecht: Reide1), p. 207.

Gilman, P.A., 1977: Geophys. Astrophys. Fluid Dyn. 3, 93.

Gilman, P.A., 1973: Seonhys. Astronhys. Fluid Dyn. 11, 157.

Gilman, P.A., 1979: Astrophys. J. (in press).

Gilman, P.A. and Foukal, P., 1979: Astrophys. ‥ 2229, 1179.

Kippenhahn, R., 1963: Astrophys. ‥ 137, 664 .

Kohler, ب.., 1970: Solar Phys. 13, 3.

Kraft, R.P., 1967: Astrophys. J. 150, 551.

Simon, G.4. and Heiss, N.0., 1953: Z. Astrophys. 69, 435.

Yoshimura, 4. and Kato, S., 1971: Publ. Astron. Soc. Janan 23, 57. 